\title{
Optical inspection of liquid crystal variable retarder inhomogeneities
}

\author{
Javier Vargas, ${ }^{1, *}$ Néstor Uribe-Patarroyo, ${ }^{1}$ Juan Antonio Quiroga, ${ }^{2}$ \\ Alberto Alvarez-Herrero, ${ }^{1}$ and Tomás Belenguer ${ }^{1}$ \\ 'Laboratorio de Instrumentación Espacial, Instituto Nacional de Técnica Aeroespacial, \\ Carretera de Ajalvir Kilometer 4, 28850 Torrejón de Ardoz, Madrid, Spain \\ ºptics Department, Universidad Complutense de Madrid, Facultad de Ciencias Físicas, \\ Ciudad Universitaria s/n, 28040 Madrid, Spain \\ ${ }^{\star}$ Corresponding author: vargasbj@inta.es
}

Received 9 September 2009; revised 20 November 2009; accepted 9 December 2009; posted 15 December 2009 (Doc. ID 116855); published 22 January 2010

\begin{abstract}
Liquid crystal variable retarders (LCVRs) are starting to be widely used in optical systems because of their capacity to provide a controlled variable optical retardance between two orthogonal components of incident polarized light or to introduce a known phase shifting (PS) between coherent waves, both by means of an applied voltage. Typically, the retardance or PS introduced by an LCVR is not homogeneous across the aperture. On the one hand, the LCVR glass substrates present a global bend that causes an overall variation of the retardance or PS. On the other hand, in the manufacturing process of an LCVR, there sometimes appears a set of micro-air bubbles that causes local retardance or PS inhomogeneities. In this work, we present an interferometric technique based on a Mach-Zehnder interferometer that is insensitive to vibrations and capable of inspecting and characterizing the LCVR's retardance or PS inhomogeneities. The feasibility of the proposed method is demonstrated in the experimental results, where the LCVR retardance is measured with an error of about $0.2 \mathrm{rad}$. The thickness of possible micro-air bubbles is obtained with a resolution of about $50 \mathrm{~nm}$. (c) 2010 Optical Society of America

OCIS codes: $\quad 120.3180,230.3720,120.4630$.
\end{abstract}

\section{Introduction}

A liquid crystal variable retarder (LCVR) is an optical device that can be used as an optical retarder (or compensator) or as a phase shifter. As an optical retarder, it provides a specific phase difference between two orthogonal components of incident polarized light. As a phase shifter, it introduces to an incident linearly polarized light ray a known phase shifting (PS) when the polarization is aligned with the slow axis of the liquid crystal (LC). In both applications, the retardance or the PS is obtained without the need of any moving mechanical parts [1-4]. These devices have other advantages, such as low mass and power consumption, that provide to this technology impor-

0003-6935/10/040568-07\$15.00/0

(C) 2010 Optical Society of America tant benefits with respect to other typical retarders or phase shifters, especially for aerospace applications. For the sake of clarity, the term "retardance" is used to denote the optical retardance and the PS unless it is needed to make the difference explicit. The change in retardation can be achieved by changing the optical thickness in a birefringence crystal, as in the case of the Soleil-Babinet compensators [5,6], using piezoelectric mirrors [6,7] or by changing the birefringence of a material, as in the case of piezoelastic modulators [6,7], Pockels/Kerr cells $[6,8]$, and LCVRs $[1-4,6]$.

An LCVR consists of an LC layer sandwiched between two flat glass plates coated with a transparent indium tin oxide (ITO) electrode and an alignment layer. The cavity formed by these plates is filled with a special blend of LCs optimized for high or low birefringence and high stability. The cell is hermetically 
sealed with glue. The alignment layer is a gently rubbed polyimide layer that is necessary for prealignment of the LC molecules. Inside the cavity, the electric field that is induced by applying a voltage on the transparent ITO electrodes modifies the alignment of the LC molecules, therefore, the retardance of the cell. Typical values of the applied voltage are in the range of $0-15 \mathrm{~V}$. Because these devices are used as highquality optical retarders, they do not have a pixelated structure (nor spacers inside the clear aperture) that produces diffraction. Instead, they are used as monolithic, monopixel devices. A diagram of an LCVR device used in this work is shown in Fig. 1(a). Figure 1(b) shows an image of an LCVR. The main disadvantages of LCVRs are their fast degradation under UV irradiation and the necessity of using a temperature control system for precise measurements.

LCVRs are theoretically unrivaled optical retarders and phase shifters for space applications because of their low mass and power consumption and because the optical retardance is obtained by means of an applied voltage, without the need of any moving mechanical parts. Additionally, LCVRs are transmission based devices, and with these systems it is possible to use large apertures. These devices have been previously analyzed under certain space conditions [9], and during 2009/2010 they will be completely space qualified under a European Space Agency project [10].

A very important parameter of an optical retarder such as an LCVR is its spatial retardance homogeneity over the full aperture. In fact, for LC modulators, the lack of spatial retardance homogeneity is often one of the main problems [11], and, therefore, this parameter has to be characterized. Other possible issues with these devices are the limited acceptance angle, transmission, or contrast and the low optical quality or retardance temperature dependence.

In the testing process of optical systems, there are many requirements on precision and accuracy that must be fulfilled. A well-established method is measuring the phase across an interference fringe pattern obtained using an interferometric approach. The principal reasons are the high phase measurement accuracy and lateral resolution, the fast measurement acquisition, and the robustness of these interferometric methods. Additionally, the phase measurement is independent of the irradiance distribution, and an accurate measurement can be obtained with low-contrast fringes. In temporal PS interferometry,

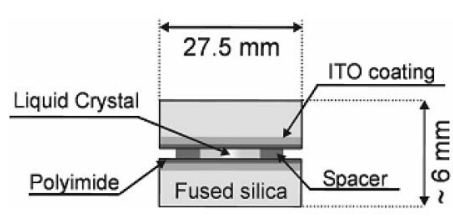

(a)

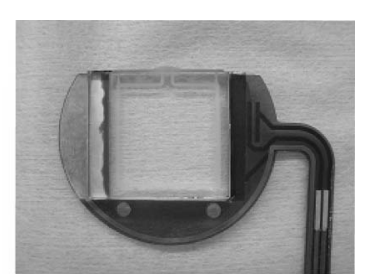

(b)
Fig. 1. (a) LCVR component diagram and (b) image of an LCVR device. three or more interferograms are obtained with a known phase shift between them [12]. A drawback of the widespread temporal PS is its susceptibility to external disturbances, such as vibration or temperature fluctuation. In many situations, the environme$\mathrm{nt}$ is the accuracy limiting factor, and sometimes the environmental conditions prevent the measurement from being performed. Different approaches have been proposed in order to reduce environmental effects. Vibration effects can be reduced by taking all the phase shifted frames simultaneously. There are several techniques to simultaneously obtain three or more phase shifted interferograms [12-16]. One possibility is to use a simultaneous PS interferometer $[13,14]$ by creating four phase shifted simultaneous interferograms captured by four CCD cameras. To reduce the effects of air turbulence, it is possible to average multiple measurements. Although simultaneous phase shift interferometers can solve the vibration effects, the use of four separate CCD cameras is troublesome because of the necessity of performing very accurate calibration and alignment procedures between the different cameras [14]. Another possibility for simultaneous PS, avoiding the multicamera system's complexity, is to use a single CCD detector into which all the phase shifted interferograms fall simultaneously [14-16]. The main drawbacks of this approach are that it is necessary to determine the corresponding detector elements between the different interferograms and that the measurement resolution is decreased.

Another perspective for reducing vibration effects in PS interferometry is to change the viewpoint from a hardware solution to a software solution. In $[17,18]$ techniques are presented for reconstructing the $\overline{\mathrm{ob}}$ ject wavefront in PS interferometry with arbitrary phase steps. These techniques can be used to perform precise and accurate PS measurements in the presence of vibration, as will be shown in the next section.

Interferometric methods, such as a polarization based interferometer [19], Mach-Zehnder interferometer [20], or holographic based techniques as in [21-23], have been used for qualitative evaluation of the flatness distortion of LC based devices and, therefore, of the retardance inhomogeneity. The objective of [19-22] is to compensate the phase distortion introduced by a pixelated LC device in order to use it in coherent optical processing techniques. These kinds of compensation are not applicable to monopixel devices, such as LCVRs, or are impractical in high-quality, diffraction-limited imaging systems in which these LCVRs are to be used. In [19], a simple method to measure the thickness uniformity of the LC layer is presented. The method is based on the birefringent nature of the LC that permits the change of the polarization state of an incident linear polarized beam. The main drawbacks of this technique are the low-contrast interferometric fringes obtained. This problem reduces the accuracy of the reconstructed phase and, therefore, the accuracy of 
the measurement. In [20], a method for using a liquid crystal television (LCTV) device in coherent optical processing techniques is shown. These types of device are affected by thickness variations over the aperture, and, therefore, their use in coherent optics is limited. In this work, a method to compensate the phase distortions introduced by the LCTV is shown. In order to remove the phase distortions, a liquid gate correction technique was used. The LCTV device was immersed in a liquid gate filled with an index matching nonconductive mineral oil. A MachZehnder interferometer was used to visually check the existence (or not) of fringes caused by phase distortions. In [21], a method to minimize the influence of the flatness distortion introduced by an LCTV without using flatness correction techniques, such as liquid gates or holographic techniques, is presented. In this work, a preliminary holographic evaluation is made to evaluate the flatness distortion introduced by the LC device. In [21], no precise quantitative information of the phase distortion introduced by the LC device is given. In [22], a low-cost holographic technique for correcting spatial phase errors of a two-dimensional LC based device is described. This technique uses the Radio Shack LCTV spatial light modulator as an optical device. The device used in [22] is characterized by its poor optical quality caused by the lack of optical flatness. In this case, the holographic interferometric pattern is used to compensate the phase distortion introduced by the LC device, but not to obtain quantitative information of the retardance inhomogeneity. In [23], two interferometric methods for measuring the thickness of nematic LC films with a free surface are presented. These methods cannot be used directly in our work, as they are not valid for obtaining measurements over the whole LCVR aperture. On the one hand, the accuracy of the proposed method in [23] seems not to be high enough, as it needs a prior measurement of parameters, such as the angle of incidence of the laser beam and the thickness and refractive index of the glass plate substrate. On the other hand, the thickness of the LC film is obtained through the measurement of the interference fringe spacing without PS techniques, which always improves accuracy.

The methods presented in [19-23] are not suitable for use in our LCVR validation study. In [19-22], the objectives of these works are to compensate the introduced phase distortion by an LC device with low optical quality, and not the precise determination of the retardance homogeneity. Our aim is to develop a highly precise and accurate, quick and fully automated method for measuring, with high spatial resolution, the retardance homogeneity over the full aperture of an elevated quantity of LCVRs (90 different LCVRs).

In this work, we present an interferometric method that is insensitive to vibrations in order to characterize the optical retardance homogeneity of LCVRs over their full aperture. This work is organized in three parts: In Section 2 , we present the theoretical founda- tions, in Section 3, experimental results are shown, and, finally, in Section $\underline{4}$, conclusions are drawn.

\section{Theoretical Foundations}

Interferometry makes use of the superposition principle to combine separate waves together in an interferogram that is diagnostic of the original state of the waves. The interferogram depends on the phase difference between waves or simply the phase. Additionally, the phase difference (or phase) depends on the optical path difference (OPD) between them that is given, for two rays coming from the same source, by

$$
\mathrm{OPD}=\left[\int_{L_{1}} n\left(s_{1}\right) \mathrm{d} s_{1}-\int_{L_{2}} n\left(s_{2}\right) \mathrm{d} s_{2}\right],
$$

where $n$ is the refractive index, and $L_{1}$ and $L_{2}$ are the path lengths crossed by the rays. From Eq. (1), the phase is given by

$$
\phi=\frac{2 \pi}{\lambda}[\mathrm{OPD}],
$$

where $\lambda$ is the wavelength. Equations (1) and (2) show that two rays traveling through the same path, but crossing different refractive indices, have a different optical path length and phase difference. This is the basis that permits characterization of materials by interferometry.

In Fig. 2, a schema of a Mach-Zehnder interferometer, used to characterize the homogeneity of a transparent sample, is shown. In Fig. 2, a Soleil-Babinet compensator is used to introduce the known phase shifts $\varphi=\left[0, \frac{\pi}{2}, \pi, \frac{3 \pi}{2}\right]$ between the two different traveling coherent beams. The phase is obtained by a typical four-step PS method.

In the presence of vibration, the values of $\varphi$ given above and introduced by the compensator are no longer valid. The effect of vibration in an interferogram is an additional time dependent unknown phase shift. The resultant intensity distribution of an interferogram affected by vibration is given by

$$
\begin{aligned}
I_{j}(x, y, t)= & I_{0}(x, y)+I_{r}(x, y) \\
& \times \cos \left[\phi(x, y)+\varphi_{j}+\delta(t)\right],
\end{aligned}
$$

where $I_{0}$ is the background intensity, $I_{r}$ is the fringe modulation, $\phi(x, y)$ is the wavefront to be measured,

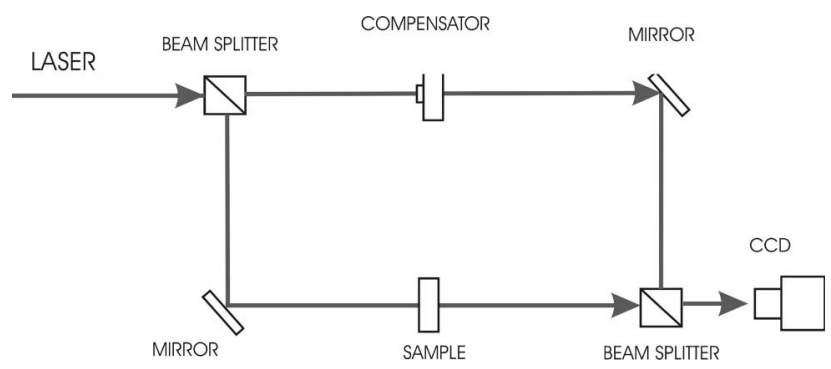

Fig. 2. Schema of the Mach-Zehnder interferometer. 


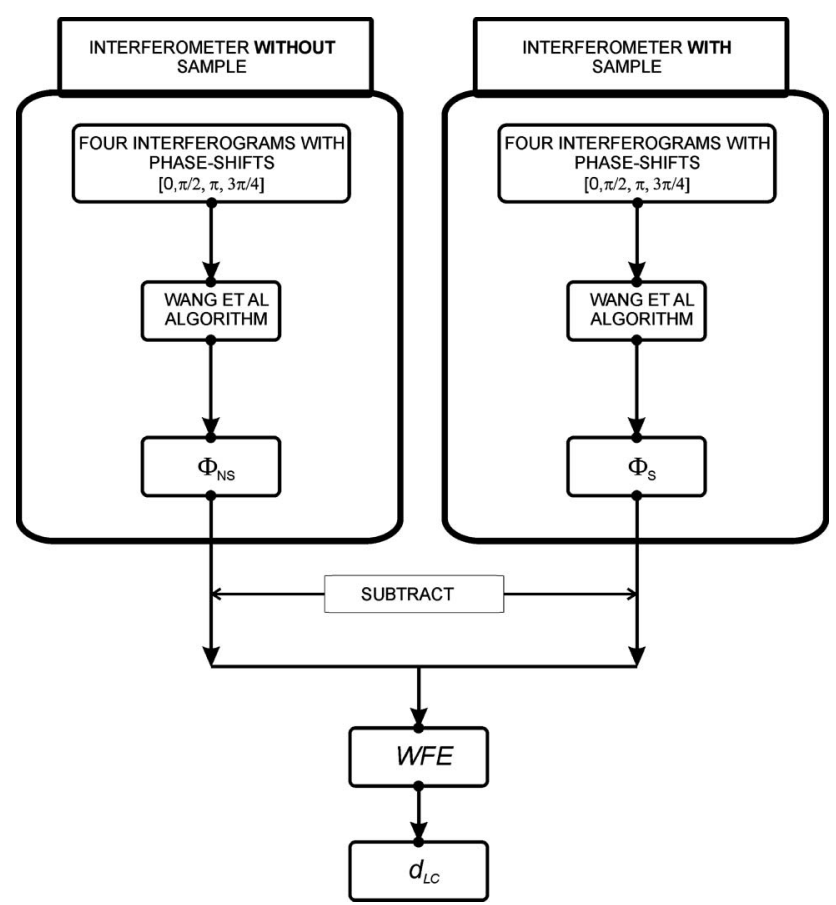

Fig. 3. Schema of the proposed measuring method.

$\varphi_{j}$ are the phase shift steps, $\delta(t)$ is the additional phase shift provoked by vibration, and $I_{j}(x, y, t)$ is the resultant fringe intensity. As can be seen from Eq. (3), it is possible to define an unknown effective phase shift step as

$$
\tilde{\varphi}_{j}(t)=\varphi_{j}+\delta(t)
$$

And then Eq. (3) is rewritten as

$$
I_{j}(x, y, t)=I_{0}(x, y)+I_{r}(x, y) \cos \left[\phi(x, y)+\tilde{\varphi}_{j}(t)\right] .
$$

Using a self-calibrating PS algorithm, as in $[17,18]$, it is possible to obtain the effective phase shifts $\tilde{\varphi}_{j}(t)$ and, therefore, an accurate measurement of $\phi(x, y)$, even in the presence of vibration. The algorithm proposed by Wang and Han [17] is based on a leastsquares iterative procedure and provides stable convergence and accurate phase extraction even if the phase shifts are completely random. The method is divided into two processes. In the first step, it is assumed that the background intensity and the modulation amplitude do not vary between interferograms. From an initial estimation of the phase steps, the phase distribution at every pixel is determined. In the second step, it is assumed that the background intensity and the modulation amplitude do not have pixel to pixel variation. Under that assumption, and from the previous phase distribution estimation obtained in the first step, the amount of phase shift in each frame is determined. The obtained phase shifts are used as inputs, and the algorithm is executed iteratively until a convergent limit is reached. In our case, the effect of the environment is expected to be low, and then an accurate initial estimation of the phase steps for the algorithm is $\tilde{\varphi}_{j}(t) \approx\left[0, \frac{\pi}{2}, \pi, \frac{3 \pi}{4}\right]$.

In the case of a sample with a homogeneous refractive index along its aperture, the resultant interferogram will be formed by a set of perfectly parallel fringes, but if the sample has a nonhomogeneous refractive index, the interferometric fringes will be deformed by Eqs. (3) and (5). Therefore, using this interferometric fringe deformation, it is possible to characterize the sample refractive index homogeneity and, therefore, the retardance homogeneity.

\section{A. Sample Refractive Index Homogeneity Characterization}

To perform a sample refractive index, homogeneity characterization is necessary in two scenarios. In the first scenario, the phase is recovered using the interferometric schema shown in Fig. 2 , but without a sample. This phase measure will be denoted by $\phi_{\text {NS }}$. In the second scenario, the phase is measured with a sample, as shown in Fig. $2\left(\phi_{\mathrm{S}}\right)$.

The phase measurement in both cases is performed using the same procedure. First, four different interferograms are obtained by introducing phase shifts of $\left[0, \frac{\pi}{2}, \pi, \frac{3 \pi}{4}\right]$ by the compensator. Once the phase measurements in both cases are obtained $\left(\phi_{S}\right.$ and $\left.\phi_{\mathrm{NS}}\right)$, the wavefront error (WFE) caused by the LCVR device is computed by subtracting them:

$$
\mathrm{WFE}=\phi_{\mathrm{S}}-\phi_{\mathrm{NS}}
$$

The LCVR retardance inhomogeneities can be determined from the root mean square (rms) of the difference between the WFE and its best fitted plane. Finally, if it is assumed that the local retardance inhomogeneities are caused by the presence of microair bubbles, the thickness of these possible micro-air bubbles inside the LC can be obtained using Eqs. (5) and ( $\underline{6})$ :

$$
\mathrm{WFE}=\frac{2 \pi}{\lambda}\left[n_{\mathrm{AIR}} d-n_{\mathrm{LC}} d_{\mathrm{LC}}-n_{\mathrm{AIR}}\left(d-d_{\mathrm{LC}}\right)\right]
$$

and then

$$
d_{\mathrm{LC}}=\mathrm{WFE} \frac{\lambda}{2 \pi\left(n_{\mathrm{AIR}}-n_{\mathrm{LC}}\right)},
$$

where $d$ and $d_{\mathrm{LC}}$ are the path length followed by a light ray in air and LC medium, respectively, $n_{\mathrm{AIR}} \cong$ 1 is the air refractive index, and $n_{\mathrm{LC}} \cong 1.3$ is the $\mathrm{LC}$ refractive index. In Eq. (8), local variations of $d_{\mathrm{LC}}$ are assumed to be caused by the presence of micro-air bubbles inside the LC layer, and then from Eq. (8), it is possible to obtain their thickness. This assumption is reasonable, as the LCVR glass substrates are typically of high quality. However, the presence of micro-air bubbles inside the LC layer is a typical issue of the LCVR manufacturing process. Figure 3 shows a schema of the explained measuring process. 


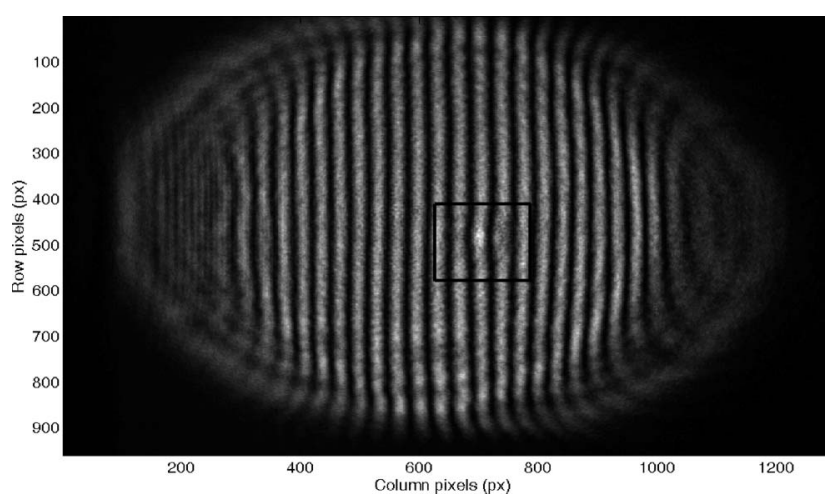

Fig. 4. One of the four interferograms obtained with the LCVR_1 sample. The rectangle shows the region of a clear fringe deformation.

\section{Experimental Results}

The measuring system is a Mach-Zehnder interferometer composed of a He-Ne laser, a USB 2.0 CCD camera with a resolution of $1280 \times 960$ pixels, and a Soleil-Babinet compensator (Thorlabs Model SBC-VIS).

The proposed method is applied to three different LCVRs, denoted LCVR_1, LCVR_2, and LCVR_3. In order to characterize the LCVRs, the WFE along the full aperture, the rms parameter, and the thickness of the LC layer $\left(d_{\mathrm{LC}}\right)$ over the full aperture are obtained using the proposed method. From the $d_{\mathrm{LC}}$ map, it is possible to estimate the thickness of possible micro-air bubbles by the local variations. Note that all the measurements are obtained without applying voltage to the LCVRs.

Shown in Fig. 4 is one of four interferograms obtained with an LCVR_1 sample. Figure 5 shows the same interferogram but without the LCV R sample. Shown in Fig. 4 is a rectangle in which the fringes are deformed in the interferogram obtained with the LCVR sample. This local fringe deformation is caused by the presence of a local inhomogeneity, which can happen with the presence of a micro-air bubble inside the LC layer. Figure 6 shows a detailed view of the fringe deformation effect of Fig. 4. In Fig. 6 , the number of fringes per millimeter has been

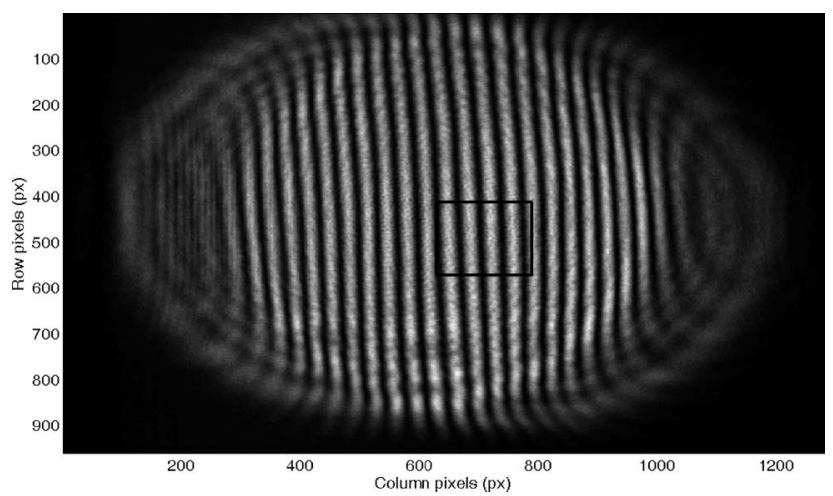

Fig. 5. One of the four interferograms obtained without the LCVR sample. The rectangle shows the region of Fig. $\underline{4}$ in which a clear fringe deformation appears.

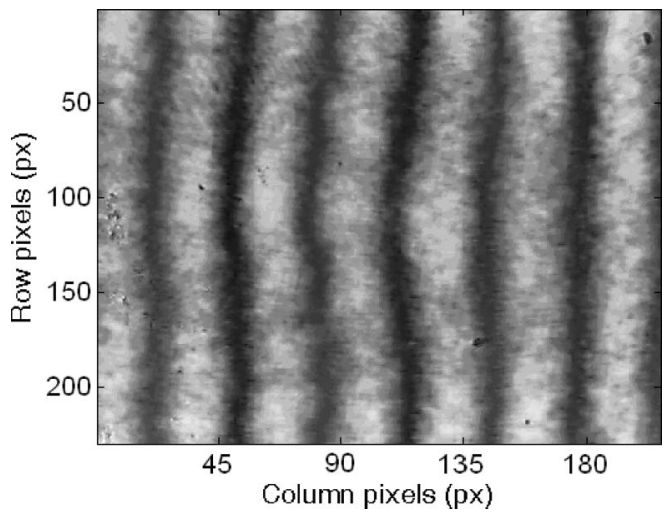

Fig. 6. Detailed view of the rectangle region in Fig. $\underline{4}$.

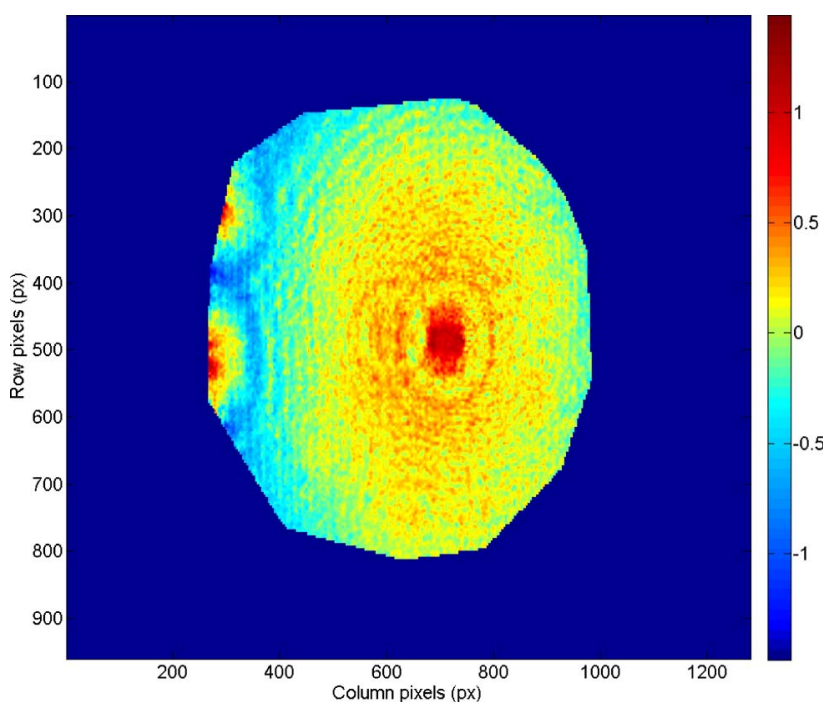

Fig. 7. (Color online) WFE measurement using the proposed method of the LCVR; its interferogram has been shown in Fig. 4 (LCVR_1).

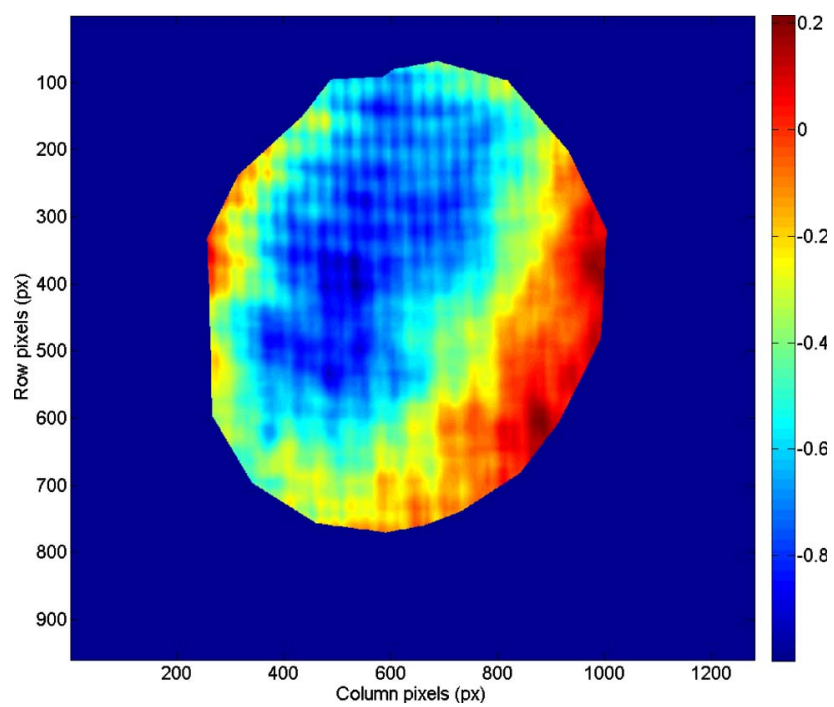

Fig. 8. (Color online) WFE measurement of the LCVR_2 sample using the proposed method. 
Table 1. Root Mean Square of the Difference Between the Wavefront Error Measurements for the LCVR Samples and Their Best Fitted Planes ${ }^{a}$

\begin{tabular}{cc}
\hline LCVR & rms (rad) \\
\hline 1 & 0.23 \\
2 & 0.18 \\
3 & 0.20 \\
\hline
\end{tabular}

${ }^{a}$ LCVR_1, 2, and 3 correspond to the WFE measurements shown in Figs. $\underline{7}-\underline{9}$, respectively.

increased with respect to Figs. 4 and 5 in order to show more clearly the fringe deformation.

Figure 7 shows an image of the WFE measurement of LCVR_1, using the proposed method, with the interferogram shown in Fig. $\underline{4}$. As can be seen from

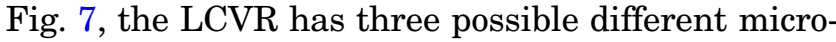
air bubbles and an overall varying retardance. Note that, here, "retardance" means an introduced PS to an incident light ray, without distinguishing between polarization states. Figures 8 and 9 show the WFE measurement of LCVR_2 and LCVR_3 samples. As can be seen from Figs. 8 and 9, there is, too, an overall varying retardance along the aperture, but there are no micro-air bubbles inside the LC layers. Table 1 shows the rms of the difference between the WFE measurements and the best fitted plane for the three LCVR samples.

Figure 10 shows a partial profile along the row of 480 pixels in Fig. 7. In Fig. 10, two of the three possible micro-air bubbles presented in Fig. 7 are clearly visible. Alternately, Fig. 10 shows the typical PS cycle error of approximately $0.2 \mathrm{rad}$. Finally, Fig. 11 shows a profile of the LC thickness for the LCVR_1 sample $\left(d_{\mathrm{LC}}\right)$ obtained from the WFE profile shown in Fig. 10, using Eq. (8). In Fig. 11, $\Delta d_{1}$ and $\Delta d_{2}$ are the measured thickness values of the two possible microair bubbles shown in the profile. Note that the thickness recovery error is about $50 \mathrm{~nm}$. In order to obtain

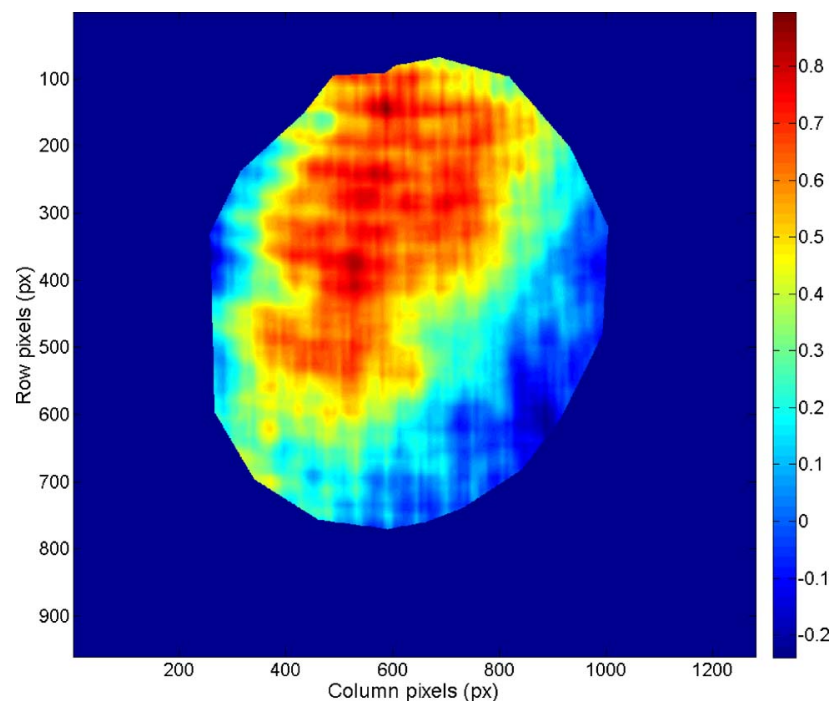

Fig. 9. (Color online) WFE measurement of the LCVR_3 sample using the proposed method.

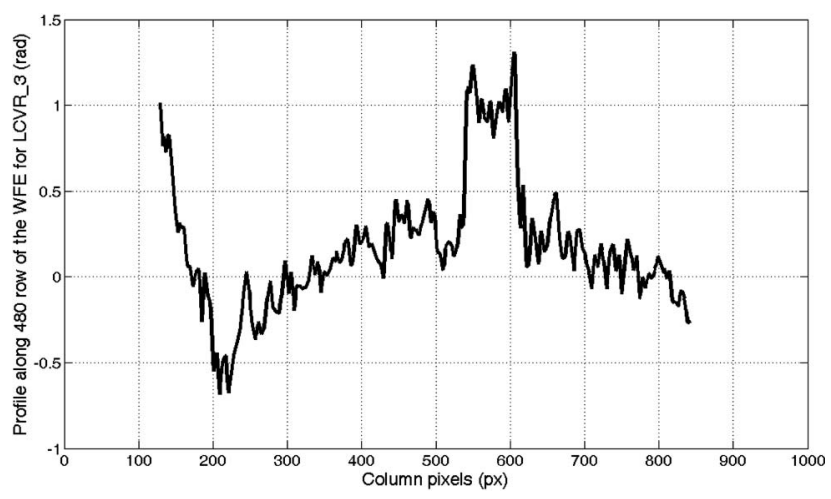

Fig. 10. Profile along row 480 of Fig. $\underline{7}$.

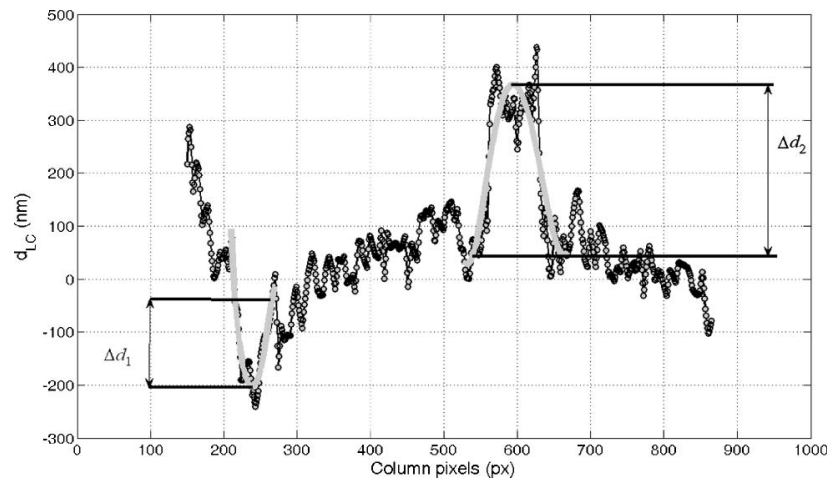

Fig. 11. Profile of the LC thickness for the LCVR_1 sample $\left(d_{\mathrm{LC}}\right)$ obtained from the WFE profile shown in Fig. 10.

Table 2. Measured Thickness Values of the Three Micro-Air Bubbles Shown in Fig. 7 for the LCVR_1 Sample

\begin{tabular}{cc}
\hline Micro-Air Bubble & $\Delta d(\mathrm{~nm})$ \\
\hline 1 & 160 \\
2 & 310 \\
3 & 120 \\
\hline
\end{tabular}

the thickness of the two visible micro-air bubbles, we have fitted them to a Gaussian profile. Table 2 shows the measured values of the thickness of these microair bubbles.

\section{Conclusions}

We proposed an interferometric method that allows us to characterize the retardance inhomogeneity of LCVR samples, among other transmission optical devices, as well as obtain the thickness of possible micro-air bubbles inside the LC layer. The proposed method is based on a typical Mach-Zehnder interferometer. The WFE is computed by a four-step PS method, improved by the use of a self-calibrating PS method to reduce environmental effects, such as vibration. With the proposed method, as has been shown in the experimental results, the WFE and the thickness of the LC are obtained with an error of about $0.2 \mathrm{rad}$ and $50 \mathrm{~nm}$, respectively. The proposed method is flexible and permits us to characterize the retardance homogeneity of a transparent 
sample in a few seconds, which is crucial for characterizing a large number of samples.

We thank the European Space Agency for economic support of this work given by the "Validation of liquid crystal variable retarders for solar orbiter polarization modulation package" project, reference number 22334/09/NL/SFe.

\section{References and Notes}

1. B. Laude-Boulesteix, A. De Martino, B. Drévillon, and L. Schwartz, "Mueller polarimetric imaging system with liquid crystals," Appl. Opt. 43, 2824-2832 (2004).

2. J. M. Bueno, "Polarimetry using liquid-crystal variable retarders: theory and calibration,” J. Opt. A: Pure Appl. Opt. 2, 216$222(2000)$.

3. L. J. November and L. M. Wilkins, "Liquid crystal polarimeter: a solid state imager for solar vector magnetic fields," Opt. Eng. 34, 1659-1668 (1995).

4. A. M. Gandorfer, "Instrumentation for optical magnetometry," in SOLMAG 2002, Proceedings of the Magnetic Coupling of the Solar Atmosphere Euroconference and International Astronomical Union Colloquium (European Space Agency, 2002).

5. M. Born and E. Wolf, "Principles of optics: electromagnetic theory or propagation," in Interference and Diffraction of Light (Cambridge U. Press, 2002).

6. B. E. A. Saleh and M. C. Teich, Fundamentals of Photonics (Wiley, 1991).

7. J. C. Kemp, "Piezo-optical birefringence modulators: new use for a long-known effect," J. Opt. Soc. Am. 59, 950-953 (1969).

8. R. W. Boyd, Nonlinear Optics (Elsevier, 2008).

9. R. L. Heredero, N. Uribe-Patarroyo, T. Belenguer, G. Ramos, A. Sanchez, M. Reina, V. Martinez Pillet, and A. ÁlvarezHerrero, "Liquid-crystal variable retarders for aerospace polarimetry applications,” Appl. Opt. 46, 689-698 (2007).

10. "Validation of liquid crystal variable retarders for solar orbiter polarization modulation package," European Space Agency, reference number 22334/09/NL/SFe. Consortium formed by the Centre Spatial de Liège, the Instituto de Astrofísica de Canarias, the Instituto de Astrofísica de Andalucía, the
Istituto Nazionale di Astrofisica, and Arcoptix. Visual display led by the Instituto Nacional de Técnica Aeroespacial.

11. F. Goudail, P. Terrier, Y. Takakura, L. Bigué, F. Galland, and V. DeVlaminck, "Target detection with a liquid-crystalbased passive Stokes polarimeter," Appl. Opt. 43, 274-282 (2004).

12. K. Creath, "Phase-measurement interferometry techniques," Prog. Opt. 34, 349-393 (2005)

13. C. L. Koliopoulos, "Simultaneous phase-shift interferometer," Proc. SPIE 1531, 119-127 (1992).

14. J. Millerd, N. Brock, J. Hayes, B. Kimbrough, M. Novak, M. North-Morris, and J. Wyant, "Modern approaches in phase measuring metrology," Proc. SPIE 5856, 14-22 (2005).

15. J. C. Wyant, "Dynamic interferometry," Opt. Photon. News 14, 36-41 (2003).

16. N. Brock, J. Hayes, B. Kimbrough, J. Millerd, M. North-Morris, M. Novak, and J. Wyant, "Dynamic interferometry," Proc. SPIE5875, 101-110 (2005)

17. Z. Wang and B. Han, "Advanced iterative algorithm for phase extraction of randomly phase-shifted interferograms," Opt. Lett. 29, 1671-1673 (2004).

18. L. Z. Cai, Q. Liu, and X. L. Yang, "Phase-shift extraction and wave-front reconstruction in phase-shifting interferometry with arbitrary phase steps," Opt. Lett. 28, 1808-1810 (2003).

19. Y. Zhisheng, L. Jifang, H. Zhengquan, and L. Yulin, "Measurements of the thickness uniformity of liquid crystal layer," Appl. Opt. 36, 9109-9110 (1997).

20. F. Mok, J. Diep, H. Liu, and D. Psaltis, "Real-time computergenerated hologram by means of a liquid-crystal television spatial light modulator," Opt. Lett. 11, 748-750 (1986).

21. N. Demoli, U. Dahms, H. Gruber, and G. Wernicke, "Influence of flatness distortion on the output of a liquid-crystaltelevision-based joint transform correlator system,” Appl. Opt. 36, 8417-8426 (1997).

22. D. Casasent and S. F. Xia, "Phase correction of light modulators," Opt. Lett. 11, 398-400 (1986).

23. Shyu-Mou Chen, Ru-Pin Pan, and Ci-Ling Pan, "Interferometric measurements of the thickness of nematic liquid crystal films with a free surface," Appl. Opt. 28, 4969-4971 (1989). 